Retracted Paper

\title{
Long Non-Coding RNA Urothelial Carcinoma Associated 1 Promotes Proliferation, Migration and Invasion of Osteosarcoma Cells by Regulating microRNA-182
}

Qiang Li ${ }^{a} \quad$ Wanying Xing ${ }^{b} \quad$ Xilong Gong $^{a} \quad$ Yueshu Wang ${ }^{a}$

${ }^{a}$ Department of Hand Surgery, China-Japan Union Hospital of Jilin University, Changchun, ${ }^{\mathrm{b} D e p a r t m e n t}$ of Breast Surgery, China-Japan Union Hospital of Jilin University, Changchun, China 